\title{
Observaciones microestructurales en el composite Al-SiC-15p obtenido por el procedimiento de compocolado ${ }^{(*)}$
}

\author{
A. Manzano-Ramírez ${ }^{(*)}$, F. Ruiz ${ }^{(* *)}$ y A. Magallanes-Castañeda ${ }^{(* * *)}$ \\ A. Manzano-Ramírez ${ }^{(*)}$, F. Ruiz ${ }^{(* *)}$ y A. Magallanes-Castañeda ${ }^{(* *)}$ \\ A. Manzano-Ramírez ${ }^{(*)}$, F. Ruiz ${ }^{(* *)}$ y A. Magallanes-Castañeda ${ }^{(* *)}$
}

Resumen El propósito del presente trabajo es estudiar el efecto de las variables del procedimiento de compocolado (compocasting), velocidad de agitación y fracción de sólido, sobre el atrapamiento mecánico o incorporación de partículas de $\mathrm{SiC}$ a la matriz de aluminio del composite Al-SiC-15p. Para ello, se variaron la velocidad de corte, $\gamma$, y la fracción de sólido $f s,\left(\gamma=27,120\right.$ y $213 \mathrm{~s}^{-1}$ y $f s=0,3,0,4$ y 0,5 , respectivamente). Se utilizó el microscopio de fuerza atómica (AFM) para observar la topografía del composite y de ello poder inferir, al menos cualitativamente, el grado de integración de las partículas de refuerzo en la matriz. Además, se emplearon las posibilidades de análisis del microscopio electrónico de barrido (SEM) para tratar de determinar la composición de la fase responsable de la adherencia matriz-partícula de refuerzo. Los resultados obtenidos en el AFM confirmaron que el atrapamiento de las partículas de refuerzo, cuando estas tienen morfología esferoidal, es más eficiente a altas fracciones de sólido $(f s>0,4)$ y elevadas velocidades de corte $\left(\gamma>120 \mathrm{~s}^{-1}\right)$, mientras que a bajas fracciones de sólido $(f \mathrm{~s}<0,3)$ y bajas velocidades de corte $\left.\gamma<27 \mathrm{~s}^{-1}\right)$ el atrapamiento es menos efectivo. Por otro lado, se presenta una evidencia cualitativa que ilustra el mecanismo de fragmentación dendrítica, consecuencia de la evolución de la estructura del sólido primario de dendrítica a globular. Finalmente, los resultados obtenidos con el SEM sugieren que la adherencia podría establecerse a través de la fase rica en silicio; sin embargo, debido al tamaño del haz de electrones, se considera que esta técnica de análisis no es la más adecuada.

Palabras clave: Compocolado (Compocasting). Atrapamiento mecánico. Microscopio de fuerza atómica. Fracción de sólido. Velocidad de corte.

\section{Microstructural observations on the composite Al-SiC-15p obtained by the compocasting process}

\begin{abstract}
In order to study how the processing variables of the compocasting process, such as shear rate and solid fraction, influence the mechanical entrapment of $\mathrm{SiCp}$ in the aluminium matrix of the $\mathrm{Al}-\mathrm{SiC}$ xxp composite. In the present work, shear rate and solid fraction were varied $\left(\gamma=27,120\right.$ and $219 \mathrm{~s}^{-1}$ and $f s=0.3,0.4$ and 0.5 , respectively). In addition, the atomic force microscope (AFM) was used to investigate the surface topography of the composite, with the purpose of determine, qualitatively, the degree of interfacial bonding between aluminium matrix and reinforcement elements. By using the chemical facilities of SEM it was attempted to determine the composition of the second phase responsible of bonding between matrix and particulate. The results obtained in the AFM confirmed that the mechanical entrapment of the particles by the solid is more efficient at high solid fractions $(f s$ $>0.4)$ and high shear rates $\left(\gamma>120 \mathrm{~s}^{-1}\right)$. In contrast, at low $f s(<0.3)$ and low $\gamma\left(<27 \mathrm{~s}^{-1}\right)$ such effect is poor. In addition, cualitative evidence, recorded by optical micrographs, illustrate the mechanism of dendritic fragmentation and change (from dendritic to globular) of the primary-solid phase in the compocasting process. SEM results suggested that bonding could be established through the silicon phase nevertheless it is considered that more accurate analysis are needed.
\end{abstract}

Keywords: Compocasting. Mechanical entrapment. Atomic force microscope. Solid fraction. Shear rate.

(•) Trabajo recibido el día 16 de febrero de 1996.

(*) CINVESTAV-IPN. Lab. de Inv. en Materiales. Centro Universitario. Cerro de las Campanas s/núm. C.P. 76010

(**) Querétaro, Qro. (México).

Instituto de Física. Universidad Autónoma de San Luis Potosí (México).

(***) Grupo Uniko. Carretera Panamericana, $\mathrm{km} \mathrm{284,} 2^{\mathrm{a}}$ Fracc. Crespo, C.P. 38000. Apdo. Postal núm. 44-A Celaya, Gto. (México).

\section{INTRODUCCIÓN}

En general, en un material compuesto se reconoce la importancia de la interfase matriz-partícula de refuerzo en lo que se refiere a las propiedades mecánicas del composite, debido, por ejemplo, al efecto de la transferencia de carga entre la matriz y 
la partícula de refuerzo. Como consecuencia de esto, es deseable una interfase con buen contacto a fin de obtener un composite con buenas propiedades mecánicas. Por otro lado, se han llevado a cabo varios trabajos con el propósito de estudiar y encontrar las mejores condiciones para una buena humectación o incorporación de la partícula de refuerzo sin provocar una excesiva reacción entre los dos constituyentes en la interfase. De esta manera, en algunos trabajos se ha observado que en la interfase se producen ciertas reacciones cuyos productos pueden degradar las propiedades mecánicas del composite (1-6), promover una buena humectabilidad de las partículas de refuerzo ( 7 y 8$)$ o formar ciertos compuestos intermetálicos que pueden ayudar a atrapar el reforzante (7). Por otra parte, el microscopio de fuerza atómica (AFM) ha resultado muy apropiado para observar y medir las características topográficas, además de permitir presentar las observaciones citadas en imágenes tridimensionales. Así, de forma general, el AFM genera imágenes de una superficie a través de un barrido que una aguja, colocada a una distancia de algunos nanómetros de distancia, efectúa a lo largo y ancho de una superficie (9).

El presente estudio, después de uno previo (10), se ha llevado a cabo con el propósito de observar hasta donde el AFM puede mostrar, cualitativamente, el grado de incorporación de partículas de refuerzo ( $\mathrm{SiC})$ a la matriz de aluminio del composite $\mathrm{Al}-\mathrm{SiC}-15 \mathrm{p}^{1}$, obtenido por el proceso de compocolado (compocasting), cuando se variaron la velocidad de corte, $\gamma$, o velocidad lineal de desplazamiento (del punto de la hélice más alejado del eje de giro) con respecto a la matriz supuesta inmóvil y no arrastrada y la fracción de sólido. Además, utilizando las facilidades de análisis químico del microscopio electrónico de barrido (SEM), se analizó la interfase matriz-refuerzo.

\section{MATERIAL Y PROCEDIMIENTO}

El equipo de reocolado que se utilizó se muestra en la figura 1. El composite ensayado era una matriz de aluminio comercial 319 con partículas de refuerzo de $\mathrm{SiC}$. El diámetro promedio de las partículas de refuerzo no recubiertas fue de $14 \mu \mathrm{m}$. Teniendo en cuenta que en el presente trabajo se decidió observar, tanto el efecto de la variación de la velocidad de corte como el de la variación de la fracción de sólido sobre el grado de incorporación de las partículas de refuerzo ( $\mathrm{SiCp}$ ), una vez alcanzados los $720{ }^{\circ} \mathrm{C}$, la potencia de la resistencia se controló para estabilizar dicha temperatura.

Posteriormente, las partículas de refuerzo, previamente precalentadas a $730{ }^{\circ} \mathrm{C}$ para eliminar humedad y evitar la pérdida excesiva de temperatu-

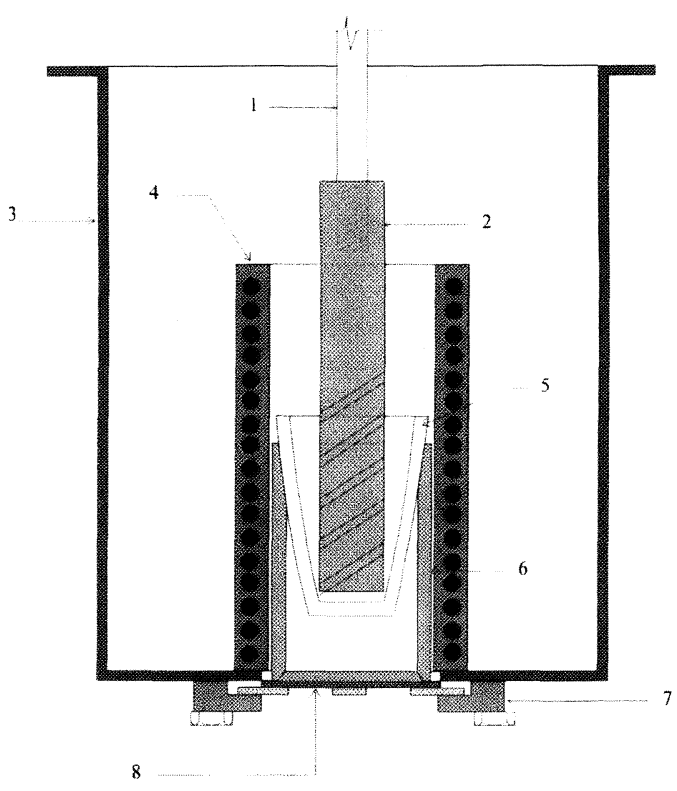

FIG. 1.- Equipo de compocolado (compocasting) utilizado.

1 - Barra de acero inoxidable de $8 \mathrm{~mm}$ de diámetro. 2 - Rotor de material cerámico (circonia), $20 \times$ $20 \mathrm{~mm}$, con un surco grabado en espiral. 3 - Carcasa de acero inoxidable de $2 \mathrm{~mm} .4$ - Resistencia eléctrica montada sobre refractario de alúmina. 5 - Crisol de alúmina. 6 - Soporte tubular de acero inoxidable. 7 - Guía de acero laminado en frío (2).

8 - Tapa de acero inoxidable.

FIG. 1.-Compocasting set up.

1 - Stainless steel bar, 8 mm diameter. 2 - Ceramic rotor (zirconia), $20 \times 20 \mathrm{~mm}$, with an helical groove. 3 - Stainless steel casing, $2 \mathrm{~mm}$. 4 - Electrical resistance on the alumina heatresistant element. 5 -Alumina crucible. 6 -Tubular stand. 7 - Cold-rolled steel guide (2). 8 -Stainless steel cover

ra en el baño metálico, fueron agregadas a dicho baño a través de un alimentador electromagnético, a una velocidad aproximada de $2 \mathrm{~g} / \mathrm{s}$, al mismo tiempo que se comenzó a enfriar la aleación y agitar continuamente hasta alcanzar las temperaturas de 590,595 y $600{ }^{\circ} \mathrm{C}$. En cada ensayo, se siguió el mismo procedimiento y se mantuvo constante la concentración de partículas de $\mathrm{SiC}$ (15\% en vol.), que se adicionaron, como se describió, por encima de la temperatura de liquidus; sin embargo, la velocidad de corte se varió a $\gamma=27 \mathrm{~s}^{-1}(100 \mathrm{rpm}), 120$ $\mathrm{s}^{-1}(450 \mathrm{rpm})$ y $213 \mathrm{~s}^{-1}$ (800 rpm).

Utilizando los procedimientos normales de metalografía, la fracción volumétrica del sólido se evaluó en muestras templadas siendo esta igual a $f s$ $=0,3\left(\right.$ para $\left.600{ }^{\circ} \mathrm{C}\right), 0,4\left(\right.$ para $\left.595^{\circ} \mathrm{C}\right)$ y 0,5 (para $590{ }^{\circ} \mathrm{C}$ ). Algunas de las muestras se examinaron con SEM, con el propósito de determinar la presencia y composición química semicuantitativa de 
segundas fases presentes en la intercara refuerzomatriz. Por otra parte, de las muestras templadas, se obtuvieron secciones de $2 \mathrm{~mm}$ de espesor con un área de $100 \mathrm{~mm}^{2}$ (utilizando una cortadora con disco de diamante) para su examen a temperatura ambiente en el AFM, que funcionó con una fuerza repulsiva constante de $10^{-18} \mathrm{~N}$ y una velocidad de barrido de $0,5 \mathrm{a} 1 \mathrm{~Hz}$.

\section{RESULTADOS Y DISCUSIÓN}

En las figuras $2 a), 2 b$ ) y 2c) se muestran las microestructuras típicas del aluminio 319 obtenido en los diferentes procesos: a) vaciado por gravedad, b) reocolado (rheocasting) y c) compocolado (compocasting). En éstas y como se esperaba, se puede ver claramente el efecto de la acción de agitación de la aleación en el estado semisólido sobre la estructura del sólido primario (Al- $\alpha$ ), ya que en lugar de la estructura dendrítica observada en la figura 2a) el sólido primario presenta una forma esferoidal después de dicha agitación (Figs. 2b) y 2c). Por otra parte, la figura 2c) muestra cómo las partículas de $\mathrm{SiC}$, agregadas a la aleación a través de vibrador electromagnético a una velocidad aproximada de $2 \mathrm{~g} / \mathrm{s}$ por encima de la temperatura de liquidus, en el composite se localizan en los espacios "interglobulares". Por otra parte, es importante mencionar que, en el caso de la microestructura mostrada en la figura $2 \mathrm{c}$ ), se observa porosidad debida a gas atrapado durante la agitación mecánica del semisólido.

Las figuras $3 a$ ), 3b) y 3c) muestran las microestructuras típicas obtenidas a $590{ }^{\circ} \mathrm{C}$ y $\gamma=27,120$ y $213 \mathrm{~s}^{-1}$, respectivamente. En éstas, se observa, cualitativamente, cómo la cantidad de $\mathrm{SiCp}$ atrapado en la matriz es más alta a medida que se incrementa la velocidad de corte. Esta misma situación se presentó para las otras fracciones de sólido. Hanumanth y Irons (11) citaron resultados similares cuando investigaron el efecto de la velocidad de mezcla sobre el contacto entre $\mathrm{SiC}$ y la matriz en una aleación de aluminio A356 con un contenido fijo de magnesio de $1,5 \%$. Este efecto se puede atribuir al hecho de que existe una mayor acción de limpieza por parte del líquido fresco sobre la superficie de las partículas. Caron y Masounave (12) sugieren que, cerca de la superficie, las fuerzas de corte que aparecen por la acción de agitación aceleran la humectación de las partículas de refuerzo. Por otra parte, trabajos previos (10) han mostrado que mediante el uso del AFM es posible obtener evidencia, al menos cualitativa, del atrapamiento del material de refuerzo. Por ello, en el presente trabajo, las características topográficas de las muestras se observaron con AFM.
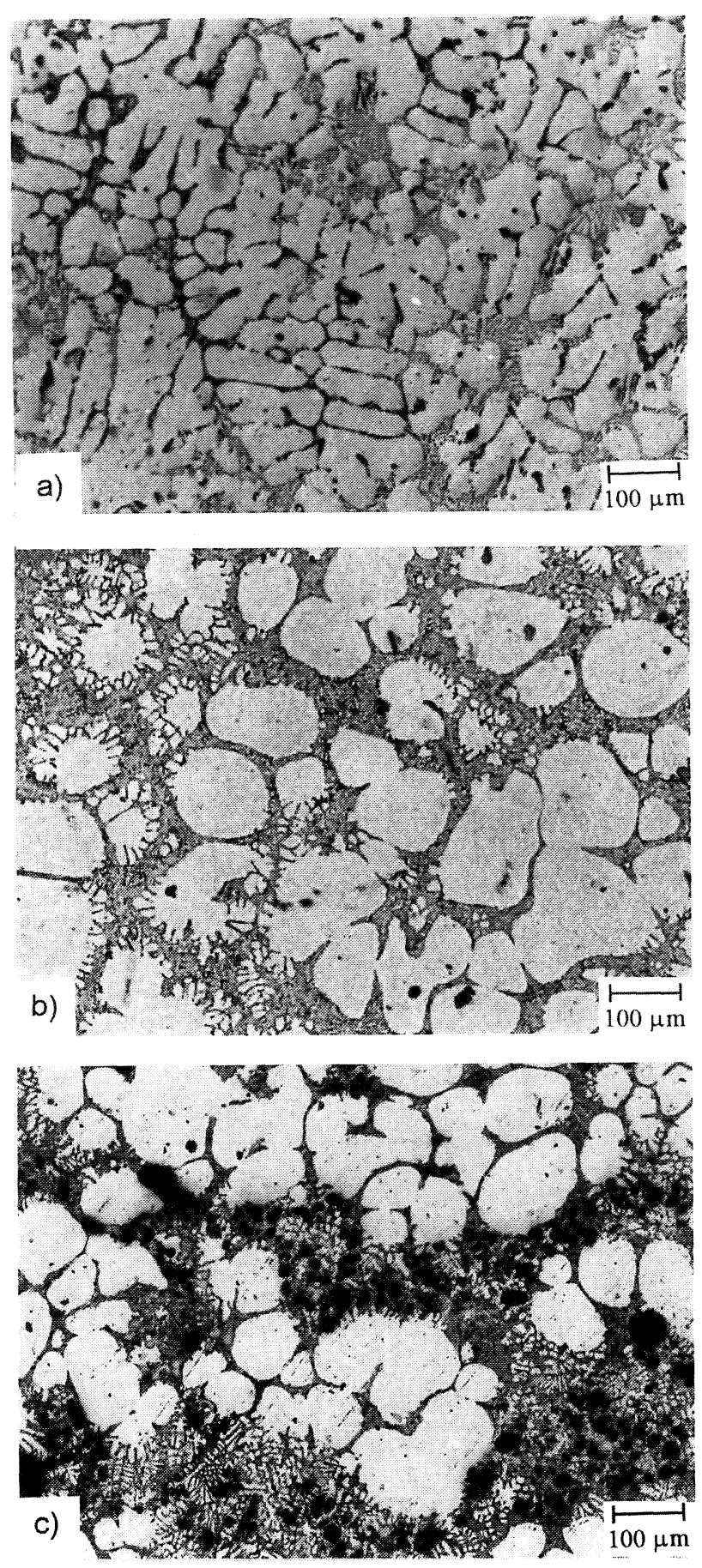

FIG. 2.- Microestructuras obtenidas de la aleación Al-319 a diferentes condiciones de solidificación: a) dendrítica, b) reocolada y templada en agua, y

c) compocolada con partículas de $\mathrm{SiC}$.

FIG. 2.- Typical microstructures of the Al-319 alloy obtained by: a) Gravity casting, b) Rheocasting, and (c) Rheocasting with reinforcement particles.

\subsection{Análisis en AFM}

En las figuras 4a), 4b) y 4c) se presenta la imagen tridimensional, la imagen de trazo y el perfil de 


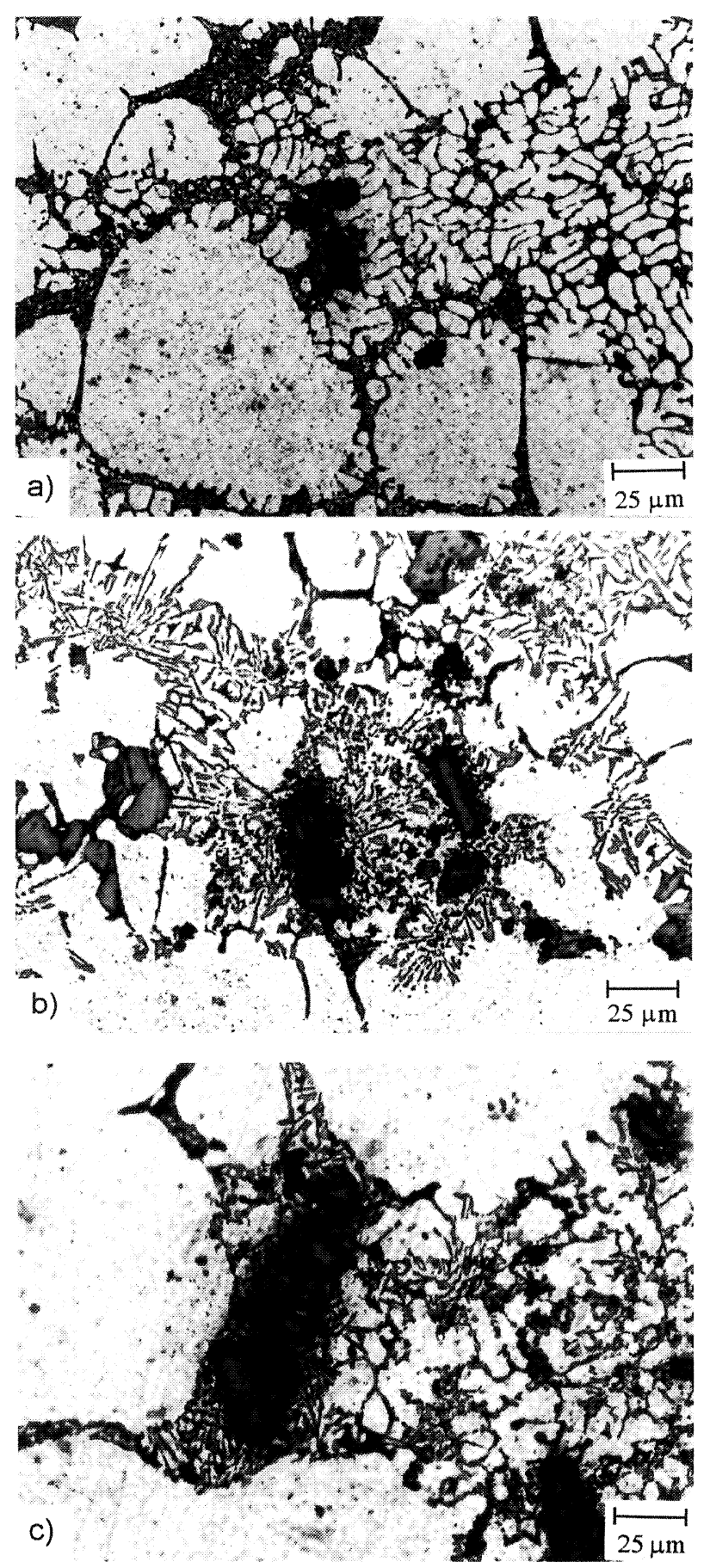

FIG. 3.- Microestructuras típicas obtenidas a 590 ${ }^{\circ} \mathrm{C}$ y: a) $\gamma=27 \mathrm{~s}^{-1}$, b) $\gamma=120 \mathrm{~s}^{-1}$, y c) $\gamma=213 \mathrm{~s}^{-1}$.

FIG. 3.- Typical microstructures of the compocast alloy with reinforcement particles obtained at $590{ }^{\circ} \mathrm{C}$ and: a) $\gamma=27 \mathrm{~s}^{-}$, b) $\gamma=120 \mathrm{~s}^{-1}$, and c) $\gamma=213 \mathrm{~s}^{-1}$.

altura de la muestra obtenida a $590{ }^{\circ} \mathrm{C}$ y $\gamma=27 \mathrm{~s}^{-1}$. Las figuras 5a), 5b) y 5c) ofrecen las mismas imágenes obtenidas para la probeta procesada a $590{ }^{\circ} \mathrm{C}$ y $\gamma=120 \mathrm{~s}^{-1}$. En las figuras $\left.\left.6 \mathrm{a}\right), 6 \mathrm{~b}\right)$ y $6 \mathrm{c}$ ) se muestran dichas imágenes obtenidas para la probeta procesada a $590{ }^{\circ} \mathrm{C}$ y $\gamma=213 \mathrm{~s}^{-1}$. De esta manera, se puede notar en las imágenes del perfil de altura (Figs. 4c), 5c) y 6c)), cambios repentinos o abruptos en las inmediaciones de las partículas de $\mathrm{SiCp}$, cambios que pueden deberse a la contracción que la matriz sufre durante la solidificación, o a errores en la detección de la topografía de la muestra por el AFM. En relación a este último punto, y sabiendo que las imágenes obtenidas en el AFM son resultado de la combinación tanto de la geometría de la punta de la aguja de detección como de la topografía de la muestra, en el presente trabajo, con objeto

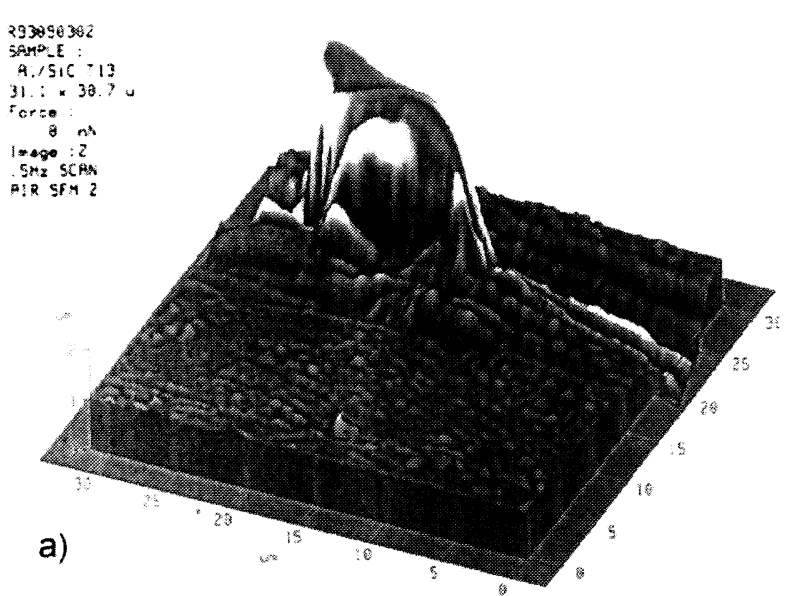

FIG. 4a).- Imagen tridimensional obtenida en el AFM de la muestra procesada a $590{ }^{\circ} \mathrm{C}$ y $27 \mathrm{~s}^{-1}$.

FIG. 4a).- $3 D$ Image obtained in the AFM from the sample processed at $590^{\circ} \mathrm{C}$ and $27 \mathrm{~s}^{-1}$.

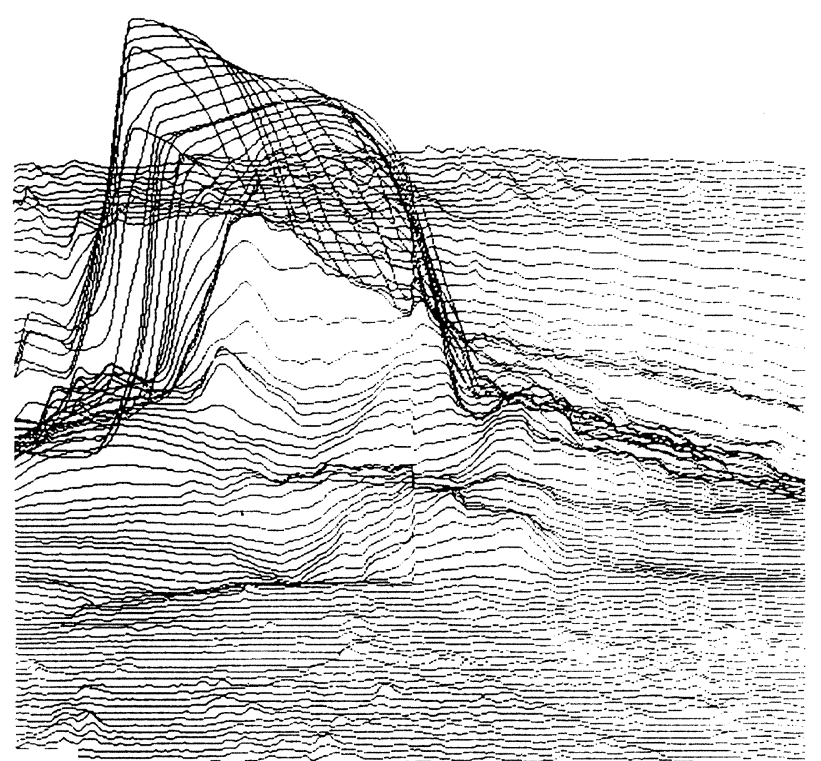

b)

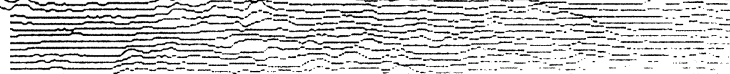

FIG. 4b).- Imagen de trazo obtenida en el AFM de la muestra procesada a $590^{\circ} \mathrm{C}$ y $27 \mathrm{~s}^{-1}$.

FIG. 4b)._- Trace image obtained in the AFM from the sample processed at $590^{\circ} \mathrm{C}$ and $27 \mathrm{~s}^{-1}$. 

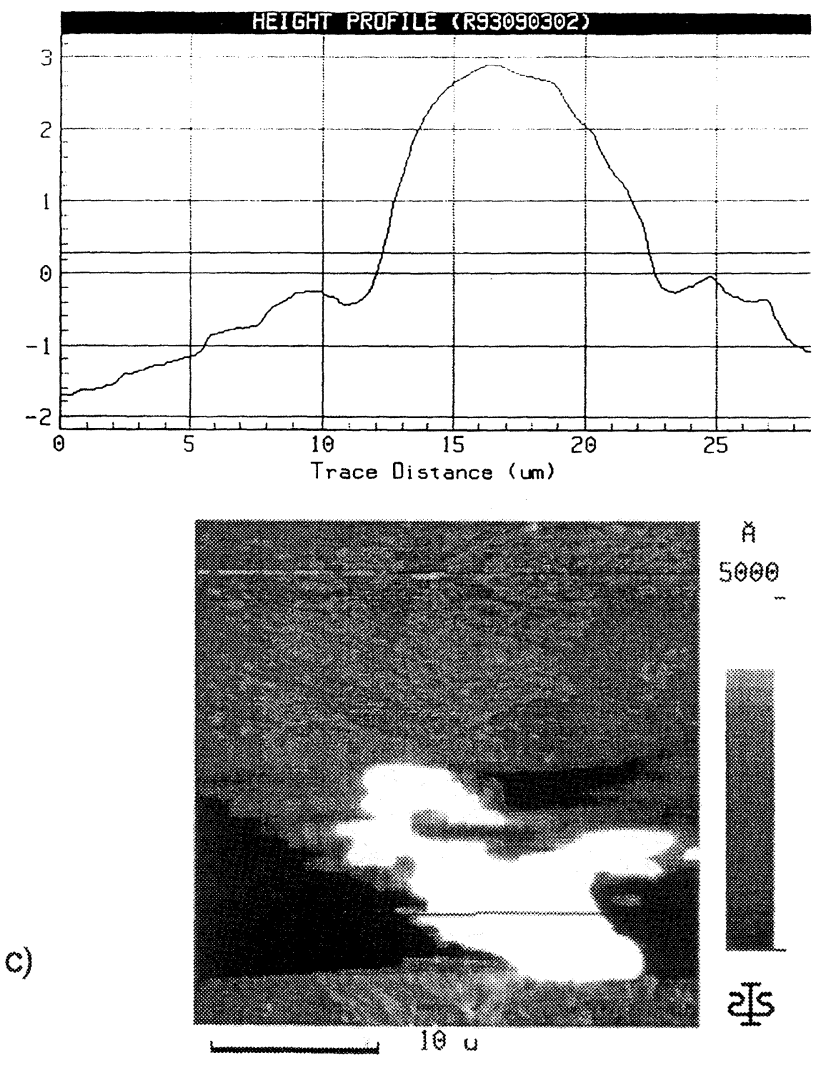

FIG. 4c).- Representación gráfica de perfil de altura obtenida en el AFM de la muestra procesada

$$
\text { a } 590{ }^{\circ} \mathrm{C} \text { y } 27 \mathrm{~s}^{-1} \text {. }
$$

FIG. 4c).- Height profile graph obtained in the AFM from the sample processed at $590{ }^{\circ} \mathrm{C}$ and $27 s^{-1}$.

de evitar errores y obtener imágenes los más cercanas a la topografía real de la muestra, se utilizó una aguja extremadamente aguda con una relación ancho-largo de 1:3; es decir, más larga que ancha, que tenía un pequeño radio de curvatura de alrede-

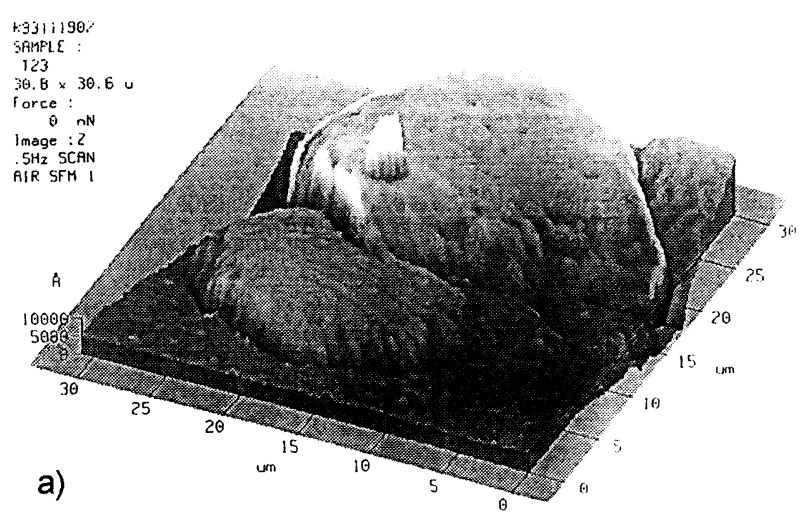

FIG. 5a).- Imagen tridimensional obtenida en el AFM de la muestra procesada a $590{ }^{\circ} \mathrm{C}$ y $120 \mathrm{~s}^{-1}$.

FIG. 5a).-3D image obtained in the AFM from the sample processed at $590^{\circ} \mathrm{C}$ and $120 \mathrm{~s}^{-1}$.

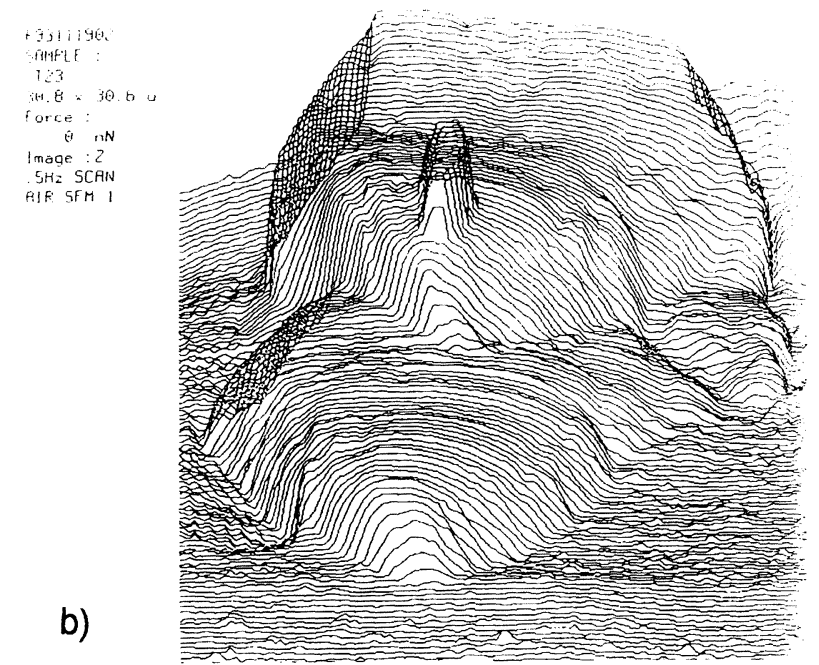

FIG. 5b).- Imagen de trazo obtenida en el AFM de la muestra procesada a $590^{\circ} \mathrm{C}$ y $120 \mathrm{~s} \mathrm{~s}^{-1}$.

FIG. 5b).- - Trace image obtained in the AFM from the sample processed at $590^{\circ} \mathrm{C}$ and $120 \mathrm{~s}^{-1}$.

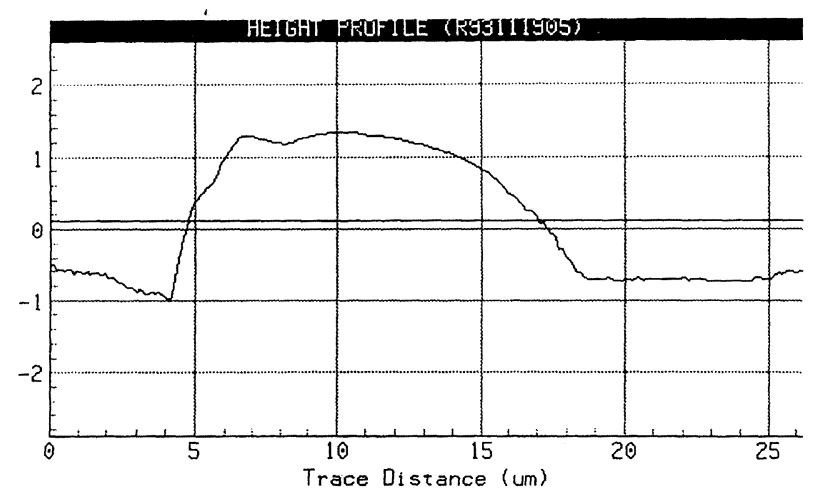

c)

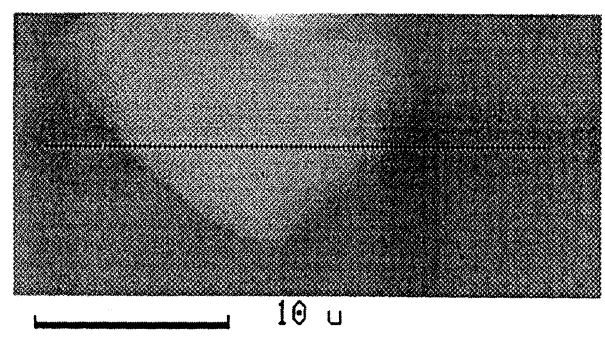

Distance: $26.33 \mathrm{um}$

FIG. 5c).- Representación gráfica de perfil de altu-ra obtenida en el AFM de la muestra procesada a $5900^{\circ} \mathrm{C} \mathrm{y} 120 \mathrm{~s}^{-1}$.

FIG. 5c).- Height profile graph obtained in the AFM from the sample processed at $590{ }^{\circ} \mathrm{C}$ and $120 \mathrm{~s}^{-1}$.

dor de 100 Ă. Debido a ello, la única fuente de los cambios repentinos o abruptos citados sería la mencionada contracción del material. Sin embargo, si éste fuera el caso, dichos cambios no tendrían por que disminuir conforme la velocidad de corte 
disminuyera, como muestran las figuras $4 \mathrm{c}), 5 \mathrm{c}$ ) y $6 c)$. De aquí que estos cambios pueden interpretarse como una mejor incorporación a altas velocidades de corte, o peor incorporación a bajas velocidades de corte. Así, de las observaciones realizadas, se puede resumir, tal y como muestra la figura 7 , el mecanismo de incorporación de partículas de refuerzo en el proceso de compocolado de la siguiente forma: en el líquido existe una baja humectación de las partículas de refuerzo debido a la existencia de un ángulo de contacto muy grande,

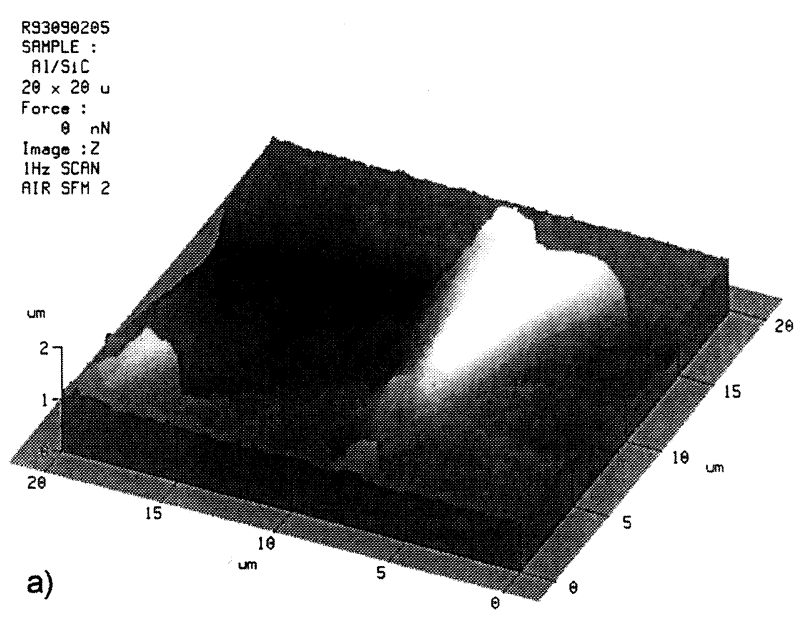

FIG. 6a).- Imagen tridimensional obtenida en el AFM de la muestra procesada a $590{ }^{\circ} \mathrm{C}$ y $213 \mathrm{~s}^{-1}$.

FIG. $6 a$ ).- $3 D$ image obtained in the AFM from the sample processed at $590^{\circ} \mathrm{C}$ and $213 \mathrm{~s}^{-1}$.

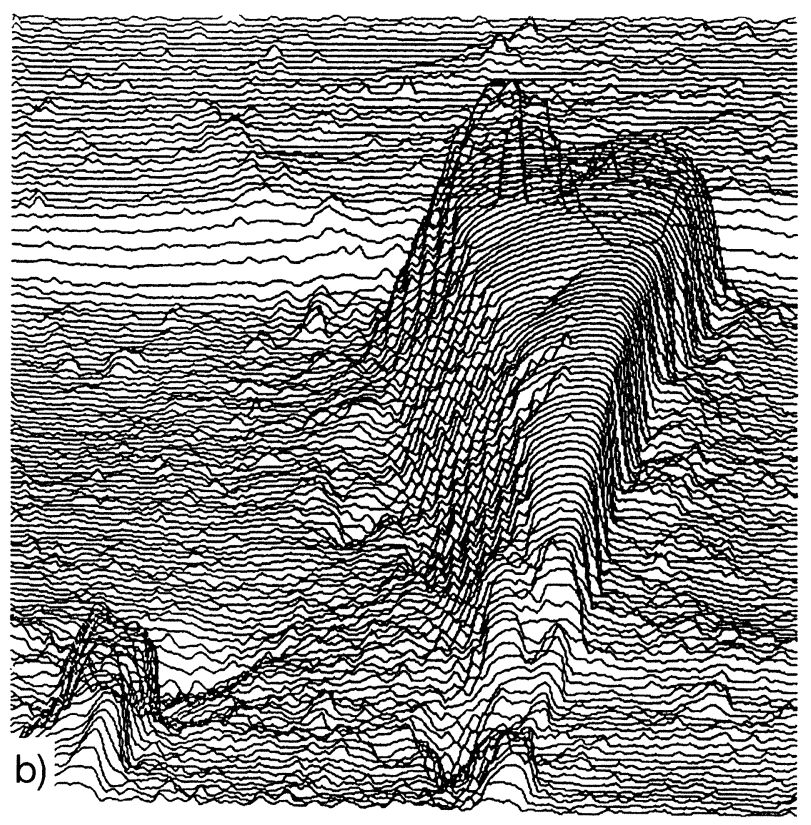

FIG. 6b).- Imagen de trazo obtenida en el AFM de la muestra procesada a $590^{\circ} \mathrm{C}$ y $213 \mathrm{~s}^{-1}$.

FIG. 6b).- Trace image obtained in the AFM from the sample processed at $590^{\circ} \mathrm{C}$ and $213 \mathrm{~s}^{-1}$.
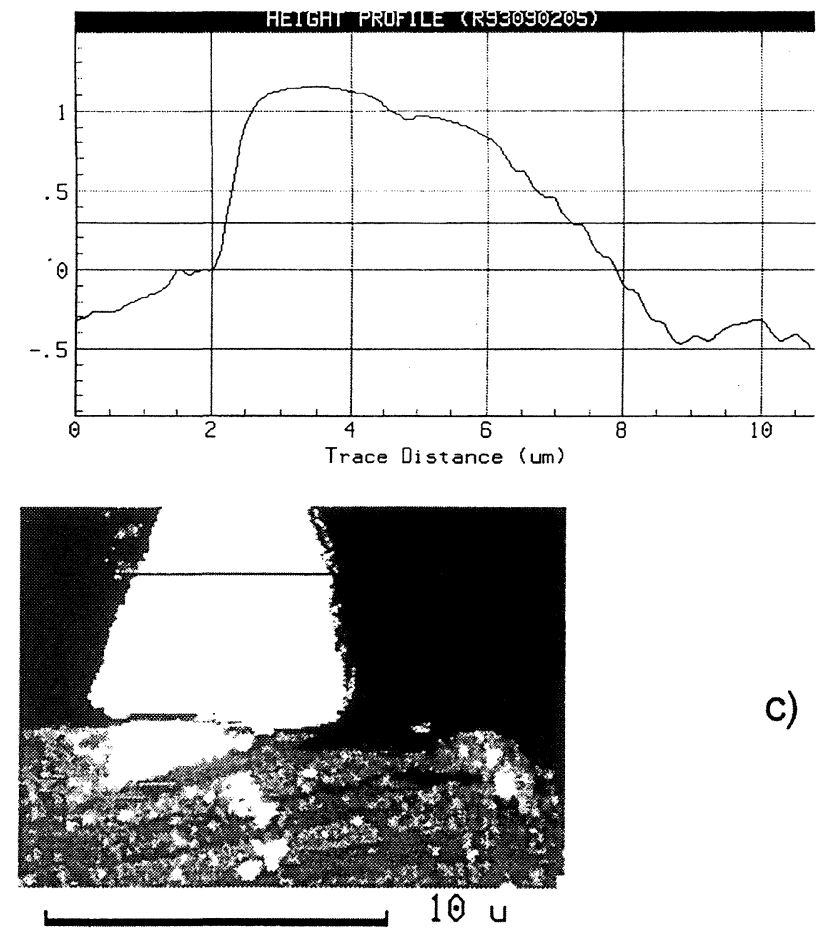

c)

Distance: 10.82 um

FIG. 6c).- - Representación gráfica de perfil de altura obtenida en el AFM de la muestra procesada a $590{ }^{\circ} \mathrm{C}$ y $213 \mathrm{~s}^{-1}$.

FIG. 6c).- Height profile graph obtained in the AFM from the sample processed at $590{ }^{\circ} \mathrm{C}$ and $213 \mathrm{~s}^{-1}$.

sin embargo, conforme la temperatura disminuye, en la región bifásica, la fracción de sólido aumenta así como lo hace la viscosidad de la aleación semisólida (14). Por ello, durante la agitación de la aleación semisólida se favorece el atrapamiento de las partículas de refuerzo (SiCp), ya que bajo el efecto de las fuerzas de corte, las partículas de sólido esferoidal "tiran" de las partículas de refuerzo hacia el interior del semisólido, aun cuando no hayan sido bien humectadas. De esta forma, queda claro que este efecto es mayor a altas velocidades de agitación o corte. Además, Caron y Masounave (12 y 15) sugieren que durante la agitación, el efecto de "lavado" sobre la superficie de la partícula es tan bueno que los óxidos dañinos o en general contaminantes pueden eliminarse, por lo que la humectabilidad de las partículas por el líquido interglobular puede ser mejorada significativamente.

\subsection{Análisis en SEM}

La figura 8 muestra el área (entre las marcas hechas con el microdurómetro) en la que se realizaron análisis semicuantitativos. Los espectrogramas del análisis semicuantitativo que se presentan 


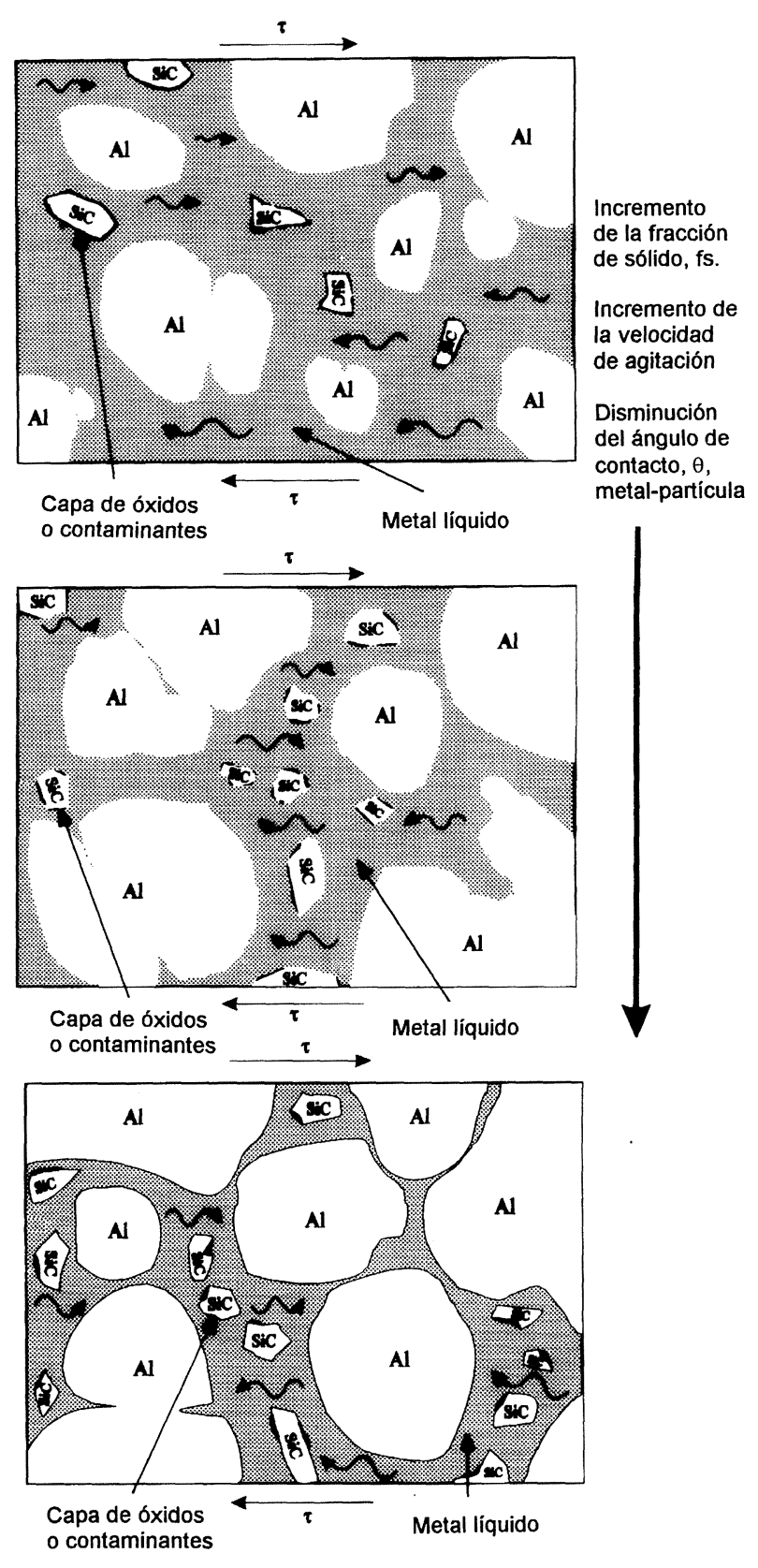

FIG. 7.- Mecanismo de atrapamiento y humectabilidad de partículas de refuerzo.

FIG. 7.-Mechanism for the entrapment and wettability of SiCp during stirring in the compocasting process.

corresponden a la muestra cuyas $\gamma=213 \mathrm{~s}^{-1}$ y $f s=$ 0,5 , debido a que estos ilustran perfectamente los resultados en las otras muestras analizadas. Así, se analizaron tres puntos: el sólido-primario esferoidal, la partícula de $\mathrm{SiC}$ y la interfase $\mathrm{SiCp}$-matriz. Los espectrogramas de dichos puntos se muestran en las figuras 9, 10 y 11, respectivamente. En éstas, se puede observar cómo, y en relación con lo esperado, la figura 9 muestra mayor concentración de aluminio, mientras que en la figura 11 se observa

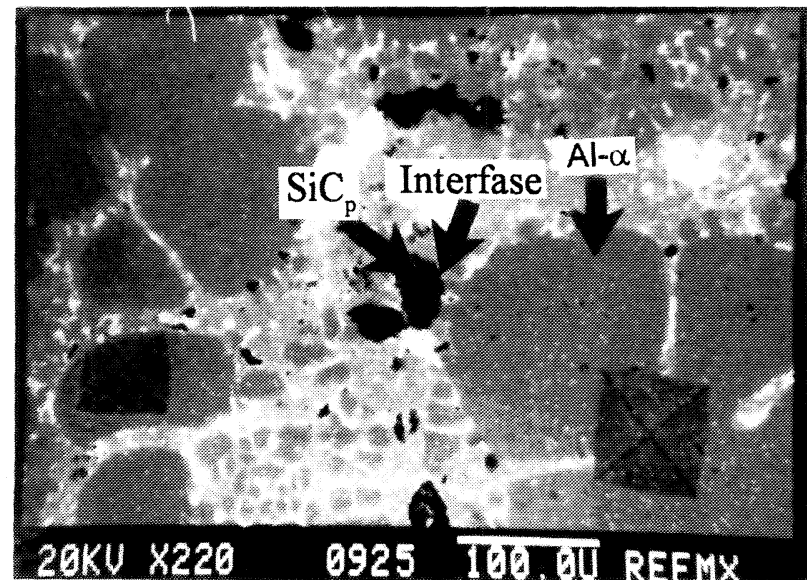

FIG. 8.- Zona en la que se realizaron los análisis químicos, mediante SEM.

FIG. 8.- Areas from which chemical analysis, by using the analytical facilities on the SEM, were carried out.

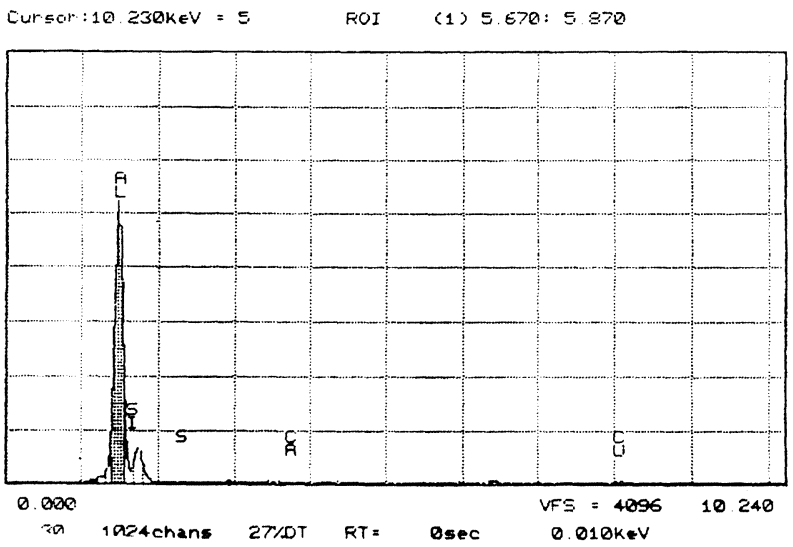

FIG. 9.- Espectrograma de análisis semi-cuantitativo realizado en una partícula de sólido primario de la muestra obtenida a $\gamma=213 \mathrm{~s}^{-1}$ y $f s=0,5$.

FIG. 9.- Semi-quantitative analysis spectra of a spheroidal particle $(A l-\alpha)$ of the sample obtained at $\gamma=213 \mathrm{~s}^{-1}$ and $\mathrm{fs}=0,5$.

mayor concentración de silicio. Por el contrario, la figura 10 muestra concentraciones similares tanto de aluminio como de silicio. Sin embargo, la concentración de silicio es mas alta que la de aluminio, por lo que se piensa que, en concordancia con lo sugerido por Pai et. al (7), la adherencia entre la matriz y la partícula puede establecerse a través de la fase silicio. No obstante y debido a las condiciones obtenidas en el SEM empleado, el diámetro del haz de electrones fue de alrededor de $10^{4} \AA$ (o 1 $\mu \mathrm{m})$. Se considera que este diámetro no fue suficientemente pequeño como para evitar abarcar parte del sólido esferoidal y/o parte de la partícula de SiC. Por lo anteriormente dicho, se estima que se 


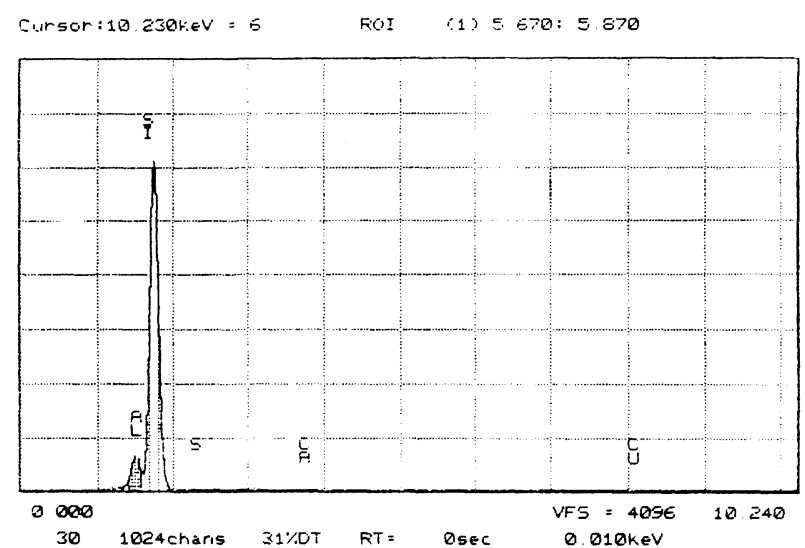

FIG. 10.- Espectrograma de análisis semi-cuantitativo realizado en una partícula de $\mathrm{SiC}$ de la muestra obtenida a $\gamma=213 \mathrm{~s}^{-1}$ y $f s=0,5$.

FIG. 10.- Semi-quantitative analysis spectra of a SiC particle of the sample obtained at $\gamma=213 \mathrm{~s}^{-1}$ and $\mathrm{fs}=0,5$.

Cursor:10.230keV = 16777215 ROI $\quad$ (1) $5.670: 5.870$

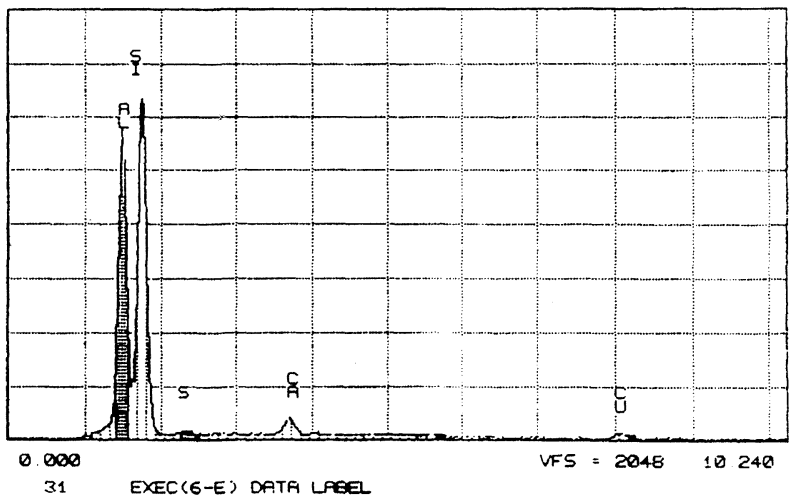

FIG. 11.- Espectrograma de análisis semicuantitativo realizado en la intercara $\mathrm{SiCp}$-matriz de la muestra obtenida a $\gamma=213 \mathrm{~s}^{-1}$ y $f s=0,5$.

FIG. 11.- Semi-quantitative analysis spectra of the interface of the SiCp-matrix of the sample obtained at $\gamma=213 \mathrm{~s}^{-1}$ and $\mathrm{fs}=0,5$.

precisan otras técnicas de análisis más exactas para definir claramente la composición de la segunda fase responsable de la adhesión en la interfase.

\subsection{Observaciones cualitativas sobre el cambio de la estructura dendrítica a esferoidal}

Después del trabajo pionero sobre el procesamiento de un semisólido (16), se han propuesto algunos mecanismos (17-21) para explicar la evolución de la microestructura de la aleación semi-sólida durante la agitación, ya que un mejor entendimiento de la evolución de dicha microestructura puede ayudar enormemente a controlar las variables de procesamiento del semisólido en el compocolado.
Todos los mecanismos señalan a la fragmentación de las dendritas del sólido primario como el hecho más relevante. Sin embargo, Doherty et. al. (18) sugieren que, antes de dicha fragmentación, en primer lugar los suaves brazos dendríticos metálicos se deforman plásticamente bajo la acción de los esfuerzos de corte generados durante la agitación para luego acumularse un gran número de defectos en la base del brazo dendrítico que se rompe por esta causa (Fig. 12). Después de esto, el fragmento comienza a ser redondeado por la acción sobre el mismo de los esfuerzos de corte. No obstante, hasta el momento no ha mostrado ninguna micrografía que ilustre el redondeamiento del fragmento dendrítico. A este respecto las figuras 13 y 14 muestran dos micrografías que los autores consideran ilustran el redondeamiento de los fragmentos dendríticos debido a la acción de los esfuerzos de corte generados durante la agitación del semisólido.

Cabe pensar que las imágenes mostradas en las figuras 13 y 14 pudieran estar condicionadas por la posición que las dendritas tienen con respecto al

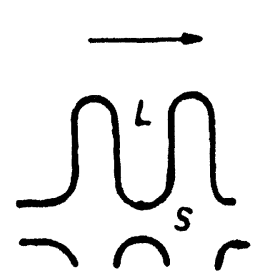

a)

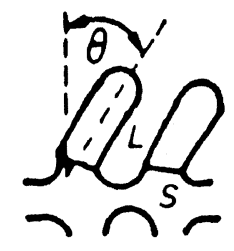

c)

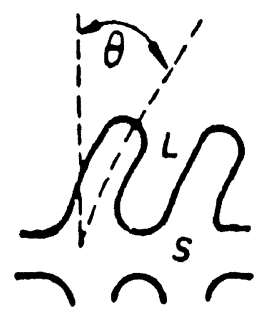

b)

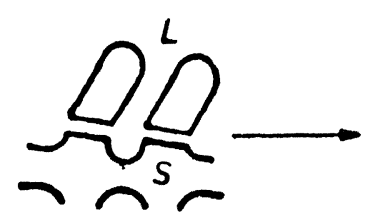

d)
FIG. 12.- Representación esquemática del modelo de fragmentación (18): a) Dendrita no deformada, b) Después de doblarse bajo la acción de los esfuerzos de corte $\tau$, c) Formación del límite de grano debido a la acumulación de dislocaciones en la base del brazo dendrítico, d) Condición $\gamma_{\mathrm{gb}}>2$ $\gamma_{\mathrm{s}-\mathrm{L}}$ por la que ocurre una completa humectación de dicho límite de grano.

FIG. 12.- Schematic representation of the model of the grain fragmentation mechanism (18): a) Undeformed dendrite, $b$ ) After bending, $c$ ) The reorganization of the lattice bending to give grain boundaries, d) For $g_{g b}>2 \gamma_{s-L}$ the grain boundaries have been "wetted" by the liquid phase. 


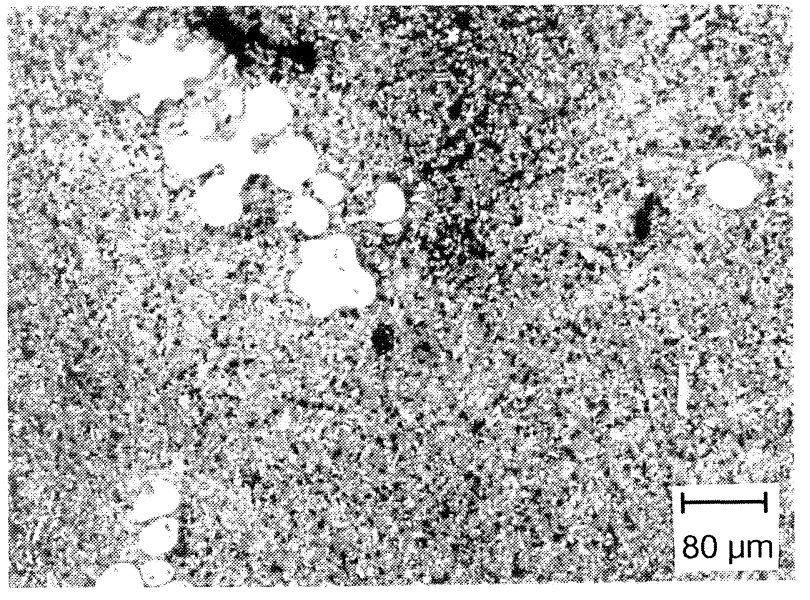

FIG. 13.- Aspecto de dendritas fragmentadas y su paulatino redondeamiento. Muestra obtenida a

$$
\gamma=213 \mathrm{~s}^{-1} \text { y } f s=0,5 \text {. }
$$

FIG. 13.-Micrograph which illustrates the dendrite fragmentation with the subsecuent appearance of spheroidal solid particles. Sample obtained at

$$
\gamma=213 \mathrm{~s}^{-1} \text { and } \mathrm{fs}=0,5 \text {. }
$$

plano de corte de la muestra durante su preparación metalográfica (Fig. 15). Sin embargo, en el presente trabajo se considera que el redondeamiento que muestran las mencionadas micrografías de los brazos dendríticos secundarios o terciarios es muy marcado; por ello, el redondeamiento no pudo depender de la posición que las dendritas tuvieran con respecto al plano de corte (22).

\section{CONCLUSIONES}

- Las observaciones realizadas con el AFM mostraron que, para las condiciones de procesamiento del composite, el grado de integración de las partículas de refuerzo en el semisólido Al-SiC$15 \mathrm{p}$ es superior a $\gamma>120 \mathrm{~s}^{-1}$ y $f_{s}>0,4$.

- En el presente trabajo, y de acuerdo con las observaciones en el AFM mencionadas, se considera que el posible mecanismo por el cual existe una mayor incorporación de las partículas de $\mathrm{SiC}$ en el semisólido durante la etapa de agitación en el proceso de compocolado, comprende los siguientes hechos importantes: el atrapamiento de las partículas de refuerzo ( $\mathrm{SiCp}$ ) por las fuerzas de arrastre, y una acción más eficiente de limpieza por líquido fresco sobre la superficie de las partículas. Ambos efectos están presentes como una consecuencia de los esfuerzos de corte que se presentan durante la agitación del semisólido.

- En el presente trabajo y de acuerdo a las observaciones realizadas, se considera que el AFM es una herramienta de trabajo adecuada y fiable

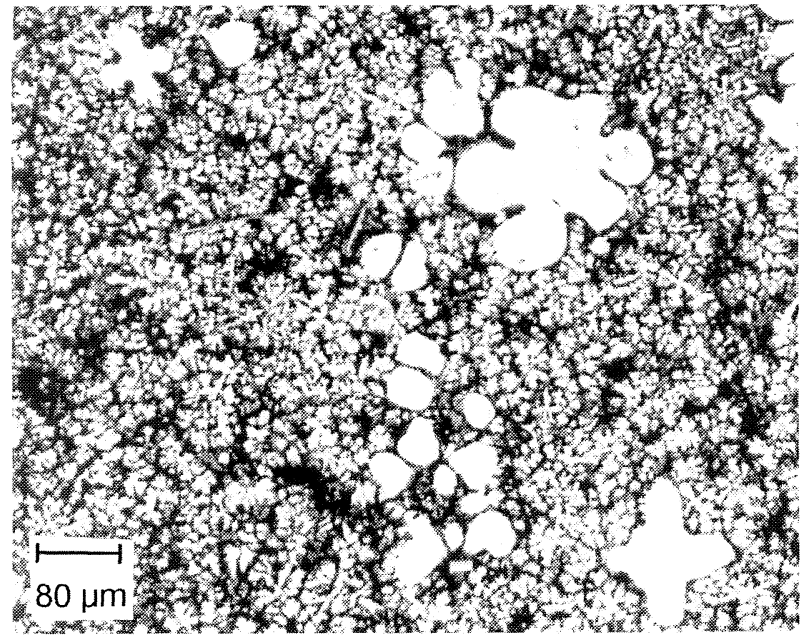

FIG. 14.- Aspecto de dendritas fragmentadas y su paulatino redondeamiento. Muestra obtenida a $\gamma=213 \mathrm{~s}^{-1}$ y $f s=0,5$.

FIG. 14.-Micrograph which illustrates the dendrite fragmentation with the subsecuent appearance of spheroidal solid particles. Sample obtained at $\gamma=213 \mathrm{~s}^{-1}$ and $\mathrm{fs}=0,5$.

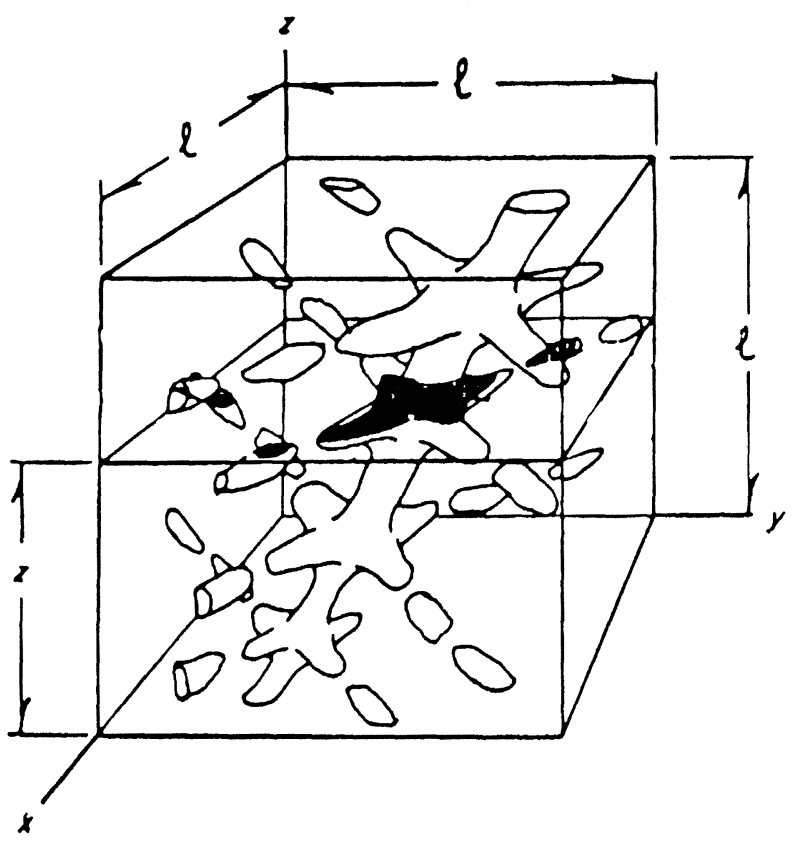

FIG. 15.- Representación esquemática (22) que ilustra la imagen que se observaría (zona sombreada) después del corte y preparación metalográfica de la muestra.

FIG. 15.- Squematic representation (22) of the image observed under the optical microscope after the metallographic preparation of the sample.

para mostrar las características topográficas de composites de matriz metálica, a partir de las cuales se puede deducir un mayor o menor grado de incorporación de las partículas de refuerzo 
en la matriz. Además, y de acuerdo con la evidencia experimental mostrada por el AFM, se considera que ello confirma que el procedimiento de compocolado es una buena alternativa para lograr la adecuada integración de las partículas de refuerzo para los contenidos de partículas estudiados.

- Los resultados con SEM obtenidos en este trabajo sugieren que la adherencia matriz-partícula de refuerzo se establece a través de una fase con silicio. Sin embargo, y debido al diámetro del haz de electrones que se estimó que se obtuvo en el presente trabajo, se considera que es necesaria otra técnica de análisis de mayor precisión.

- A partir de la evidencia experimental mostrada en el presente trabajo, se refuerza la validez de la teoría del mecanismo de fragmentación dendrítica del sólido primario y el redondeamiento de éste, ambos debidos a los esfuerzos de corte generados durante la agitación del semisólido.

\section{Agradecimiento}

Los autores agradecen a COSNET y CONACYT el apoyo económico para la realización del presente trabajo, así como al Ing. Eruviel Cordova Rivera su ayuda en la preparación de las muestras.

\section{REFERENCIAS}

(1) Metcalfe, A.G., "Interfaces in Metal Matrix Composites”, Cap. 6, Academic Press. Nueva York, 1974: 269.

(2) Kannikeswaren, K. y Lin, R.Y. J. Met., 1987: 17-19.

(3) Llyod, D.J. y Chamberland, B. "Cast Reinforced Metal Composites”. ASM Proc. Conf. Chicago, IL (EE.UU.), 1988: 263-271.

(4) LEUCHT, R., DUDEK, H.J. y ZIEGIER, G. Zwerstofftech, 18, 1987: 27.

(5) Jarfors, A.E.W., SvendSEn, L., Wallinder, M. y FreDRIKSSON, H. Metall. Trans. A, 24A, 1993: 2.577-2.583.
(6) Legoux, J.G., L’Esperance, G., SAlvo, L. y SuÉry, M. Proc. Conf. Fabrication of Particulates Reinforced Metal Composites. Ed. J. Masounave y F.G. Hamel. ASM Intern. Materials Park, OH (EE.UU.), 1990: 31.

(7) Pai, B.C., Pillai, S.G.K., Pillai, R.M. y Satyanarayana, K.G. Proc. Conf. Solidification of Metal Matrix Composites. Ed. Pradeep Rohatgi. TMS Warrendale, PA (EE.UU.), 1989: 193.

(8) Ghosh, P.K. y RaY, S. Proc. Conf. Fabrication of Particulates Reinforced Metal Composites. Ed. J. Masounave y F.G. Hamel. Quebec (Canadá), Sep., 1990: 23.

(9) Artifacts in SPM. TopoMetrix Corporation. Santa Clara, CA (EE.UU.), 1993.

(10) Manzano, A., Nava, E. y Vázquez, C. Scr. Metall. Mater., 29, 1993: 1.241-1.246.

(11) Hanumanth, G.S. y Irons, G.A. Proc. Conf. Fabrication of Particulates Reinforced Metal Composites. Montreal, Quebec (Canadá), Sep. 1990: 41-46.

(12) Caron, S. y Masounave, J. Ibíd: 107-112.

(13) Geiger, A.L. y Walker, J.A. JOM, 43 (8), 1991: 8.

(14) Flemings, M.C. Metall. Trans. B, 22B (3), 1991: 269293.

(15) Caron, S. y Masounave, J. Proc. Conf. Fabrication of Particulates Reinforced Metal Composites. Montreal, Quebec (Canadá), Sep. 1990: 79-86.

(16) Spencer, D.B., Merabian, R. y Flemings, M.C. Metall. Trans., 3, 1972: 1.925-1.932.

(17) Vogel, A. Metall. Sci., 12, 1978: 576-578.

(18) Lee, H.I., Doherty, R.D., Feest, E.A. y Titchmarsh. Proc. Conf. Solidification and Cast Metals. Sheffield (R.U.), 1977: 119-125.

(19) Doherty, R.D., Lee, H.I. y FeEst, E.A. Mater. Sci. Eng., 65, 1984: 181-189.

(20) Suery, M. Proc. Conf. Semi-Solid Processing of Alloys and Compocasting. CEMEF. Sophia-Antipolis (Francia), 4-6 Abr. 1990,

(21) Jabrane, S., Clément, B. y Ajersch, F. Proc. Conf. Processing of Semi-Solid Alloys and Composites. MIT. Cambridge, MA (EE.UU.), 10-12 Jun., 1992: 223-236.

(22) Sellars, C.M. Quantitative Metallography Notes. Sheffield Univ. Dep. of Metallurgy. Sheffield (R.U.), 1986: 6. 